\title{
HARD POLARIZED PHOTON EMISSION IN SINGLE CRYSTALS BY HIGH ENERGY ELECTRONS FOR PLANAR CRYSTAL ORIENTATIONS $\theta$
}

\author{
S.M.Darbinyan ${ }^{a}$ and N.L.Ter-Isaakyan ${ }^{b}$ \\ Yerevan Physics Institute Yerevan 375036, Armenian \\ ${ }^{a}$ E-mail: simon@lx2.yerphi.am \\ ${ }^{b}$ E-mail: terisaak@jerewan1.yerphi.am
}

\begin{abstract}
The radiation emission spectra of polarized photons emitted from charge particle in single crystal are obtained in semiclassical theory in Baer-Katkov-Strakhovenko approximation for planar crystal orientation. The range of applicability of this approximation is estimated by comparing the results with calculations in exact semiclassical theory. Optimal crystal orientations for producing unpolarized and polarized photon beams are also founded.
\end{abstract}

1. Introduction. It is well known that the processes of photon emission from charged particles (as well as $e^{-} e^{+}$pair creation by photon) at high energies in oriented single crystals reveal very strong angular and energy dependence. The photon emission spectra in crystals in their maxima could exceed the corresponding values for amorphous target 100 and more times and the radiation appears essentially polarized. Therefore, these processes are widely applied in experimental physics for the production of high energy polarized photon beams, as well as for the analyses of photon polarization. For incident angles $\vartheta$ to crystal axis/planes $\vartheta \gg \vartheta_{v}$, where $\vartheta_{v}$ is the characteristic angle given by $\vartheta_{v}=U_{0} / m\left(U_{0}\right.$ is the scale of ax$\mathrm{ial} /$ planar potential and $m$ the electron mass) these processes are well described in the theory of coherent bremsstrahlung (CB) and pair production (CPP) [1]. This theory is constructed in the framework of the first Born approximation in crystal potential and fails at small angles, where these processes become of magnetic bremsstrahlung nature.

In the papers of V.N. Bayer V.M. Katkov and V.M. Strakhovenko has been developed a general theory of basic QED processes in strong crystalline fields (see i.e. new English edition of their book [2]). This theory is not restricted by the first Born approximation and is based on the semiclassical character of motion of ultrarelativistic particles in strong fields. However, the numerical calculations in the exact theory face serious mathematical difficulties and some approximation and modeling methods have been developed. First numerical results of the radiation emission spectra in the semiclassical theory were published in [3] (in the framework of additional modeling assumption); exact calculations were presented in [4]. In Ref. [5] an analytical method of calculations in the framework of semiclassical approach was developed and new numerical results were presented. The radiation emission spectra of polarized photons in semiclassical approach were calculated in $[2,6]$ and first numerical results were presented in our previous paper [6]. These papers demonstrate an essential difference of exact spectra from the corresponding results of coherent theory at small angles $\vartheta_{0} \leq \vartheta_{v}$. There is a good agreement between first experimental results at small angles $[7,8]$ and these calculations.

Polarization characteristics of radiation emission in single crystals in the region of applicability of $\mathrm{CB}$ are well investigated in experiments (at the energies of incident electrons up to $10 \mathrm{GeV}$ ) and the $\mathrm{CB}$ theory describes the experimental results satisfactory[1].

\footnotetext{
${ }^{1}$ The work is supported by ISTC grant A-099
} 
2. Main results. In this paper we have derived the polarized photon emission spectra in the framework of Bayer-Katkov-Strakhovenko approximation of paper [3] (further BKS approximation) which is valid at small incident electrons angles to one of crystallographic planes. We also modified the nonpolarized equations of [3] and present all results in the form very convenient for numerical calculations. Our calculation algorithm allows to reduce the computing time of all polarized spectra to 5-10 sec (Pentium 233) depending on accuracy and crystal orientation.

Comparing the calculation in BKS approximation [4] with exact calculations in semiclassical theory we estimated the range of applicability of this approximation. It appears, that the equation in BKS approximation described the exact spectra satisfactory only at very small angles of incidence, about 3-5 times less than critical angle of planar channeling.

We present a large number of polarized emission spectra in wide range of angles for different energies and crystal orientations. The analysis of these results make it possible to find optimal crystal orientations for producing high energy photon beams. For producing nonpolarized high energy photons it seems optimal to use the range of hard photon peaks at large $x$, where $x$ is a fraction of electron energy $\varepsilon$ carried by emitted photon. The maximum of intensity we find for $<110>$ oriented crystal for incident electron moving close to (111) or (110) planes. However, the degree of polarization at hard photon peaks appeared to be rather low. We find that the degree of polarization at given $x$ achieves its possible maximal value ( $\sim 55 \%$ at $x=2 / 3$, at $\varepsilon=200 \mathrm{GeV}$ ) at relatively large values of incident angles to crystal axes, when the corresponding hard peak situated at given $x$ is of pure coherent nature. The optimal configurations for producing polarized high energy photons we find for $<001>$ oriented diamond crystal when electron moves close to (110) plane and for $\langle 110\rangle$ oriented crystal when electron also moves close to (11̄0) plane.

3. Polarized photon spectrum in BKS approximation. Let us start from the general semiclassical formula for polarized photon spectrum [2.6], which we present in the following form in terms of Stocks parameters $\xi_{1}, \xi_{2}, \xi_{3}$ :

$$
\frac{d I}{d \omega}=\frac{\alpha}{\pi} \frac{m^{2} \omega}{\varepsilon^{2}} \int \frac{d^{3} r}{V} F(\mathbf{r}, \vartheta)\left[\int_{0}^{\infty} \frac{d \tau}{\tau}\left[\left(T_{0}+\gamma^{2} \xi_{1} T_{1}+\gamma^{2} \xi_{3} T_{3}\right) \sin A_{1}+\gamma^{2} \xi_{2} T_{2} \cos A_{1}\right]-\frac{\pi}{2}\right]
$$

Here $\varepsilon$ and $\omega$ are electron and photon energies, $\gamma=\varepsilon / m ; \mathbf{r}$ is the entry point, $V$ is crystal volume, integration over $\mathbf{r}$ gives the averaging over electron trajectories in crystal, $F(\mathbf{r}, \vartheta)$ is the coordinate distribution function at given value of $\vartheta$. Eq.1 must be also averaged over time $t$. The quantities of $A_{1}=A_{1}(\tau)$ and $T_{i}=T_{i}(\tau), i=0,1,2,3$, in (1) in rectilinear trajectory approximation have the following form:

$$
\begin{gathered}
A_{1}=\frac{m^{2} \omega \tau}{\varepsilon \varepsilon^{\prime}}\left[1+\sum_{\mathbf{q}, \mathbf{q}^{\prime}} \frac{G(\mathbf{q}) G\left(\mathbf{q}^{\prime}\right)}{m^{2} q_{z} q_{z}^{\prime}} \mathbf{q}_{\perp} \mathbf{q}_{\perp}^{\prime}\left(\frac{\sin \left(\left(q_{z}+q_{z}^{\prime}\right) \tau\right)}{\left(q_{z}+q_{z}^{\prime}\right) \tau}-\frac{\sin \left(q_{z} \tau\right) \sin \left(q_{z}^{\prime} \tau\right)}{q_{z} q_{z}^{\prime} \tau^{2}}\right) e^{-i\left(\mathbf{q}+\mathbf{q}^{\prime}\right) \mathbf{r}(t)}\right] \\
T_{0}=1-\varphi(\varepsilon) \sum_{\mathbf{q}, \mathbf{q}^{\prime}} \frac{G(\mathbf{q}) G\left(\mathbf{q}^{\prime}\right)}{m^{2} q_{z} q_{z^{\prime}}} \mathbf{q}_{\perp} \mathbf{q}_{\perp}^{\prime} \sin \left(q_{z} \tau\right) \sin \left(q_{z}^{\prime} \tau\right) e^{-i\left(\mathbf{q}+\mathbf{q}^{\prime}\right) \mathbf{r}(t)}, \\
T_{1}=-\sum_{\mathbf{q}, \mathbf{q}^{\prime}} \frac{G(\mathbf{q}) G\left(\mathbf{q}^{\prime}\right)}{m^{2} q_{z} q_{z}^{\prime}}\left(q_{x} q_{y}^{\prime}+q_{y} q_{x}^{\prime}\right)\left[g\left(q_{z} \tau\right) g\left(q_{z}^{\prime} \tau\right)+\sin \left(q_{z} \tau\right) \sin \left(q_{z}^{\prime} \tau\right)\right] e^{-i\left(\mathbf{q}+\mathbf{q}^{\prime}\right) \mathbf{r}(t)}
\end{gathered}
$$




$$
\begin{gathered}
T_{2}=\varphi(\varepsilon) \sum_{\mathbf{q}, \mathbf{q}^{\prime}} \frac{G(\mathbf{q}) G\left(\mathbf{q}^{\prime}\right)}{m^{2} q_{z} q_{z}^{\prime}}\left(q_{x} q_{y}^{\prime}-q_{y} q_{x}^{\prime}\right)\left[g\left(q_{z} \tau\right) \sin \left(q_{z}^{\prime} \tau\right)-g\left(q_{z}^{\prime} \tau\right) \sin \left(q_{z} \tau\right] e^{-i\left(\mathbf{q}+\mathbf{q}^{\prime}\right) \mathbf{r}(t)},\right. \\
T_{3}=-\sum_{\mathbf{q}, \mathbf{q}^{\prime}} \frac{G(\mathbf{q}), G\left(\mathbf{q}^{\prime}\right)}{m^{2} q_{z} q_{z}^{\prime}}\left(q_{x} q_{x}^{\prime}-q_{y} q_{y}^{\prime}\right)\left[g\left(q_{z} \tau\right) g\left(q_{z}^{\prime} \tau\right)+\sin \left(q_{z} \tau\right) \sin \left(q_{z}^{\prime} \tau\right)\right] e^{-i\left(\mathbf{q}+\mathbf{q}^{\prime}\right) \mathbf{r}(t)}, \\
g(x)=\frac{\sin x}{x}-\cos x .
\end{gathered}
$$

The notation used here coincides, in general, with ones of Ref.[2]. The crystal potential is used in the form of $U(r)=\sum G(\mathbf{q}) e^{-i \mathbf{q r}}$, where $\mathbf{q}$ is reciprocal lattice vector; $\varepsilon^{\prime}=\varepsilon-\omega$, $\varphi(\varepsilon)=\frac{\varepsilon^{\prime}}{\varepsilon}+\frac{\varepsilon}{\varepsilon^{\prime}}, \mathbf{r}(t)=\mathbf{r}+\mathbf{v}_{0} t$. The Stocks parameters and projections of vectors correspond to the frame defined by following unit vectors:

$$
\hat{e}_{x}=\left[\mathbf{n}_{2} \mathbf{v}_{0}\right], \hat{e}_{y}=\mathbf{n}_{2}, \hat{e}_{z}=\mathbf{v}_{0},
$$

where $\mathbf{v}_{\mathbf{0}}$ is the incoming electron velocity, $\mathbf{n}_{\mathbf{2}}$ is a unit vector transverse to $\mathbf{v}_{\mathbf{0}}$ and to the main crystallographic axis.

Let $\vartheta$ be the electron incident angle to the given crystallographic axes and $\psi$ be electron incident angle to the given crystallographic plane, then $\sin \psi=\sin \vartheta \sin \varphi$, where $\varphi$ is the angle of $\mathbf{v}_{0}$ projection onto the plane perpendicular to the given crystallographic axis, to the given crystallographic plane.

The BKS approximation is valid for planar crystal orientations under the following conditions.

1. $\varphi \ll 1$, this makes it possible to isolate the continuous planar potential contribution splitting the sums in (2-6) into two parts, $\sum_{\mathbf{q}}=\sum_{\mathbf{q}}^{F}+\sum_{\mathbf{q}}^{w}$, where the sum $\sum_{\mathbf{q}}^{F}$ contains $\mathbf{q}$ for which $q_{z} \rightarrow 0$ when $\varphi \rightarrow 0$ and the sum $\sum_{\mathbf{q}}^{w}$ includes all other terms.

2. $\psi \ll 1, \psi$ should be small enough, so that $\left|q_{z}\right| \tau \ll 1$, this makes it possible to expand the functions in the sum $\sum_{\mathbf{q}}^{F}$ in powers of $\left|q_{z}\right| \tau$.

3. $\vartheta$ should not be too small, $\vartheta \gg \vartheta_{v}$ so that the sum $\sum_{\mathbf{q}}^{w}$ be small enough and Eqs.2-6 could be expanded in powers of $\sum_{\mathbf{q}}^{w}$.

Two last conditions crucially depend on the effective upper integration limit over $\tau$ in (1), $\tau_{0}$. The value of $\tau_{0}$ could not be exactly established from general considerations due to rather complicated dependence of Eq.2 on $\tau$, so, further we try to estimate the range of validity of BKS approximation by comparing the BKS results with calculations in exact semiclassical theory.

Following the steps of Ref.3, we did the above mentioned expansions, performed time averaging and integrated the results over $\tau$. All polarized intensity spectra we succeeded to present via one fold integrals of known special functions:

$$
\frac{d I}{d \omega}=\frac{\alpha}{\pi} \frac{m^{2} \omega}{\varepsilon^{2}} \int \frac{d y}{d_{p l}} F(y, \vartheta)\left(I_{0}+\xi_{1} I_{1}+\xi_{2} I_{2}+\xi_{3} I_{3}-\frac{\pi}{2}\right) \text {, }
$$


where the integration is taking over coordinate $y$ perpendicular to the plane, $d_{p l}$ is the distance between the planes. Circular polarization vanishes in BKS approximation, $I_{2}=0$. The quantities $I_{0}, I_{1}, I_{3}$ are defined as follows:

$$
\begin{aligned}
& I_{0}=F_{0}(z, \lambda)+\beta \chi^{2} F_{2}(z, \lambda)+ \\
& \sum^{w} \frac{G^{2} q_{\perp}^{2}}{m^{2} q_{z}^{2}}\left\{\left(\frac{\beta}{4}+\frac{u}{s^{2}}\left(z-\frac{8 u\left(\chi \mathbf{q}_{\perp}\right)^{2}}{s^{2} q_{\perp}^{2}}\right)\right)\left(2 F_{0}(z, \lambda)-F_{0}\left(z_{+}, \lambda\right)-F_{0}\left(z_{-}, \lambda\right)\right)-\right. \\
& \frac{u}{s}\left(F_{0}\left(z_{+}, \lambda\right)-F_{0}\left(z_{-}, \lambda\right)\right)+\frac{u^{2} \chi^{2}}{s^{2}}\left(1-\frac{2\left(\chi \mathbf{q}_{\perp}\right)^{2}}{\chi^{2} q_{\perp}^{2}}\left(1-\beta\left(1+\frac{\rho}{2}\right)+\frac{4 \beta \chi^{2}}{s^{2}}\right)\right) 2 F_{2}(z, \lambda)- \\
& \frac{u^{2} \chi^{2}}{s^{2}}\left(1+\frac{2\left(\chi \mathbf{q}_{\perp}\right)^{2}}{\chi^{2} q_{\perp}^{2}}\left(1-\beta\left(1+\frac{\rho}{2}\right)-\frac{4 \beta \chi^{2}}{s^{2}}\right)\right)\left(F_{2}\left(z_{+}, \lambda\right)+F_{2}\left(z_{-}, \lambda\right)\right)- \\
& \frac{u \chi^{2} \beta}{s^{2}}\left[\left(1-\frac{2\left(\chi \mathbf{q}_{\perp}\right)^{2}}{\chi^{2} q_{\perp}^{2}}\right) 2 F_{1}(z, \lambda)-\left(1+\frac{2\left(\chi \mathbf{q}_{\perp}\right)^{2}}{\chi^{2} q_{\perp}^{2}}\right)\left(F_{1}\left(z_{+}, \lambda\right)+F_{1}\left(z_{-}, \lambda\right)\right)\right]- \\
& \left.\frac{8 u^{2}\left(\chi \mathbf{q}_{\perp}\right)^{2}}{s^{3} q_{\perp}^{2}}\left(1-\beta\left(1+\frac{\rho}{2}\right)\right)\left(F_{1}\left(z_{+}, \lambda\right)-F_{1}\left(z_{-}, \lambda\right)\right)\right\} \\
& I_{1}=\sum_{\mathbf{q}}^{w} \frac{G^{2} q_{x} q_{y}}{m^{2} q_{z}^{2}}\left\{\frac{z^{2}}{s^{2}}\left(2 F_{0}(z, \lambda)-F_{0}\left(z_{+}, \lambda\right)-F_{0}\left(z_{-}, \lambda\right)\right)+\right. \\
& \left.\frac{u \chi^{2}}{s^{2}}\left[3\left(2 F_{1}(z, \lambda)-F_{1}\left(z_{+}, \lambda\right)-F_{1}\left(z_{-}, \lambda\right)\right)+2 z F_{2}(z, \lambda)-z_{+} F_{2}\left(z_{+}, \lambda\right)-z_{-} F_{2}\left(z_{-}, \lambda\right)\right]\right\} \\
& I_{3}=\chi^{2} F_{2}(z, \lambda)+ \\
& \sum_{\mathbf{q}}^{w} \frac{G^{2} q_{\perp}^{2}}{m^{2} q_{z}^{2}}\left\{\frac{2 u\left(\chi \mathbf{q}_{\perp}\right)^{2}}{s^{2} q_{\perp}^{2}}\left[2 F_{2}(z, \lambda)\left(z-\frac{4 u \chi^{2}}{s^{2}}\right)+\left(F_{2}\left(z_{+}, \lambda\right)+F_{2}\left(z_{-}, \lambda\right)\right)\left(z+\frac{4 u \chi^{2}}{s^{2}}\right)\right]-\right. \\
& \frac{u \chi^{2}}{s^{2}}\left[\left(1-\frac{2\left(\chi \mathbf{q}_{\perp}\right)^{2}}{\chi^{2} q_{\perp}^{2}}\right) 2 F_{1}(z, \lambda)-\left(1+\frac{2\left(\chi \mathbf{q}_{\perp}\right)^{2}}{\chi^{2} q_{\perp}^{2}}\right)\left(F_{1}\left(z_{+}, \lambda\right)+F_{1}\left(z_{-}, \lambda\right)\right)\right]+ \\
& \left.\frac{8 u\left(\chi \mathbf{q}_{\perp}\right)^{2} z}{s^{3} q_{\perp}^{2}}\left(F_{1}\left(z_{+}, \lambda\right)-F_{1}\left(z_{-}, \lambda\right)\right)\right\}+ \\
& \sum_{\mathbf{q}}^{w} \frac{G^{2}\left(q_{y}^{2}-q_{x}^{2}\right)}{2 m^{2} q_{z}^{2}}\left\{\frac{z^{2}}{s^{2}}\left(2 F_{0}(z, \lambda)-F_{0}\left(z_{+}, \lambda\right)-F_{0}\left(z_{-}, \lambda\right)\right)-\right. \\
& \left.\frac{u \chi^{2}}{s^{2}}\left[2 F_{1}(z, \lambda)-F_{1}\left(z_{+}, \lambda\right)-F_{1}\left(z_{-}, \lambda\right)-2 z F_{2}(z, \lambda)+z_{-} F_{2}\left(z_{+}, \lambda\right)+z_{+} F_{2}\left(z_{-}, \lambda\right)\right]\right\}
\end{aligned}
$$

where

$$
\begin{aligned}
\chi & =\frac{i \varepsilon}{m^{3}} \sum_{\mathbf{q}}^{F} G(\mathbf{q}) \mathbf{q} \mathbf{e}^{-i q_{y} y} \\
\frac{\rho}{2} & =\sum_{\mathbf{q}}^{w} \frac{|G(\mathbf{q})|^{2} q_{\perp}^{2}}{m^{2} q_{z}^{2}}
\end{aligned}
$$


$u=\omega / \varepsilon^{\prime}, \quad s=2 \varepsilon q_{z} / m^{2}, \quad \lambda^{2}=\chi^{2} u, \beta=\varphi(\varepsilon), \quad z=u(1+\rho / 2), \quad z_{ \pm}=u(1+\rho / 2 \pm s / u)$. The functions $F_{0}(z, \lambda), F_{1}(z, \lambda)$ and $F_{2}(z, \lambda)$ could be expressed via known special functions. We present and analyze them in Appendix. The nonpolarized intensity spectra, $I_{0}$, coincide with result of Ref.[2], if we take for $F_{0}(z, \lambda)$ the integral representation via Bessel functions (Eq.7 of $[2])$.

\section{Numerical calculation results and conclu-} sions.In this section we present the numerical calculations for uniform distributions, keeping, of course, in mind, that for real spectrum one should take into account the particle flux redistribution $\left(F\left(y, \theta_{0}\right) \neq 1\right)$ for $\psi \leq \psi_{c}$, where $\psi_{c}$ is the critical angle of planar channeling, $\psi_{c}=\sqrt{\frac{2 U_{0}}{\varepsilon}}, U_{0}$ is the depth of planar potential well. For $<001>$ orientation we define the angle $\varphi$ as the angle of the projection of $v_{0}$ onto the plane (001) to the plane $(1 \overline{1} 0)$, similarly, for $<110>$ orientation the angle $\varphi$ is the angle of the projection of $v_{0}$ onto the plane (110) to the plane (001). The typical intensity spectrum and Stocks parameters are presented on Fig. 1 for $<110>$ oriented diamond crystal. Note, that throughout the paper we present all angles in Radian. The soft part of the spectrum is completely determined by magnetic bremstrahlung contribution. There is a wide polarization maximum of essential linear polarization $\left(\xi_{1}=0, \xi_{3} \sim 0.5-0.7\right)$ in wide range of $x \leq 0.3$. The shape of intensity and polarization spectra in soft region are not changed by the variation of the angle $\varphi$, because in BKS approximation the continuous planar potential contribution was actually derived in the limit $\varphi \rightarrow 0$. The high frequency peaks in hard part of the spectra could be interpreted as coherent peaks slightly modified by influence of continuous planar potential.

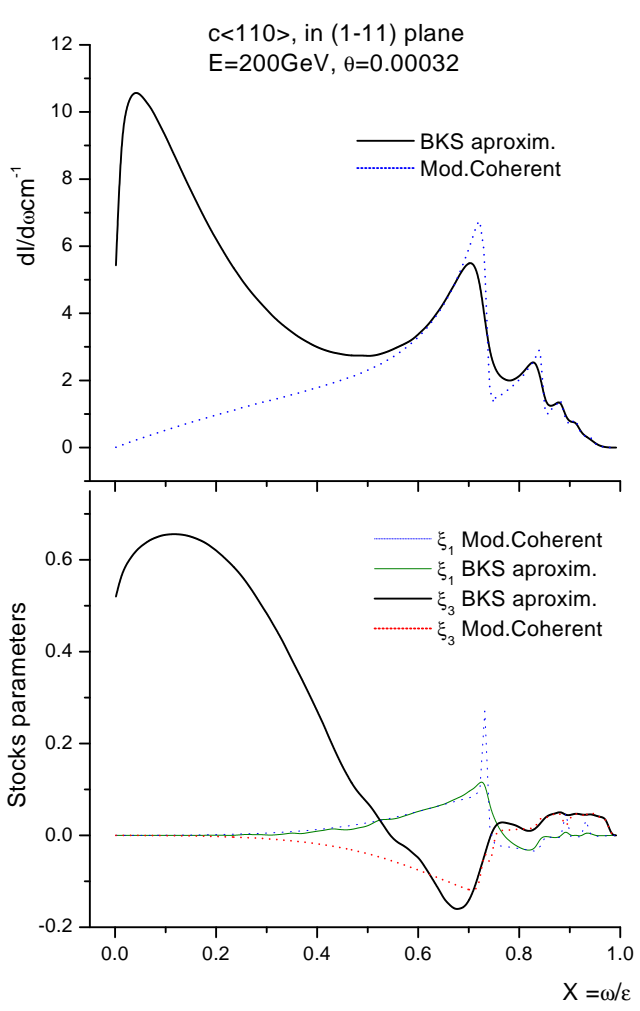

Fig.1: Comparison the hard part of spectral distributions of intensity and Stocks parameters in modified coherent and semiclassical (BKS) approaches This region can be approximately described by modified coherent theory [2], if we exclude from the sums the reciprocal vectors corresponding to magnetic bremstrahlung contribution $\left(q_{z} \rightarrow 0\right.$ when $\left.\varphi \rightarrow 0\right)$. It is demonstrated on Fig.1. In such approximation each hard peak corresponds to the sum of coherent contributions of reciprocal vectors, so that for each peak corresponding values of $q_{z}$ tend to the fixed limit at $\varphi \rightarrow 0$ [6]. The polarization in hard region strongly depend on crystal orientation and will be discussed later.

In order to estimate the range of applicability of BKS approximation we also perform exact semiclassical calculations (making use of Eq.1-6) at different incident angles to the plane 

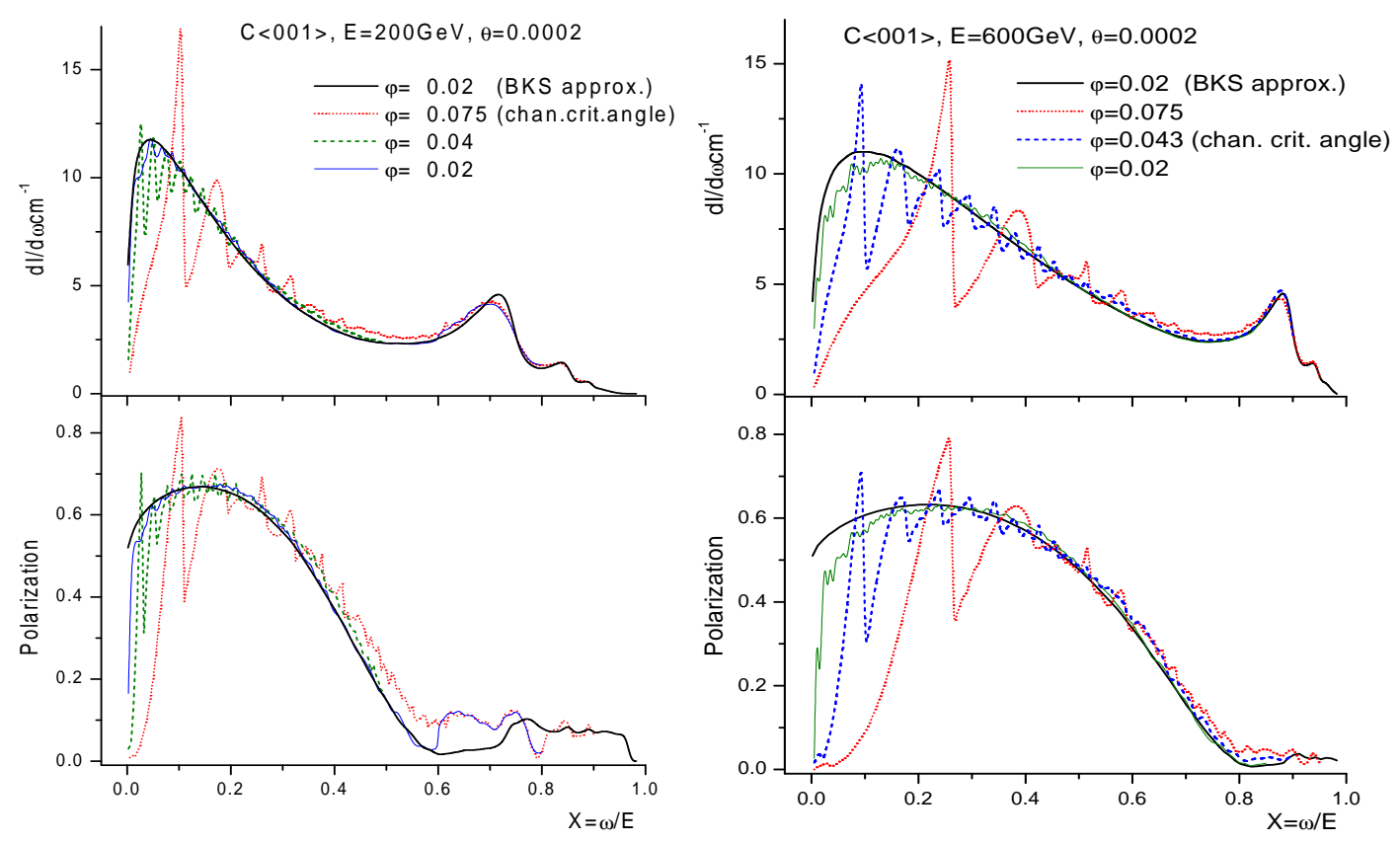

Fig.2: Comparison the spectral distributions of intensity and degree of polarization of BKS approximation with exact semiclassical calculations.

for different energies. To carry out the arising integrals over time of complicated oscillating function we have elaborated special integration program. Results are presented in Fig.2. It is very interesting that when electron incident angle to the plane is equal or even less that planar channeling critical angle, $\psi_{c}$, the soft part of the spectrum strongly differ from BKS

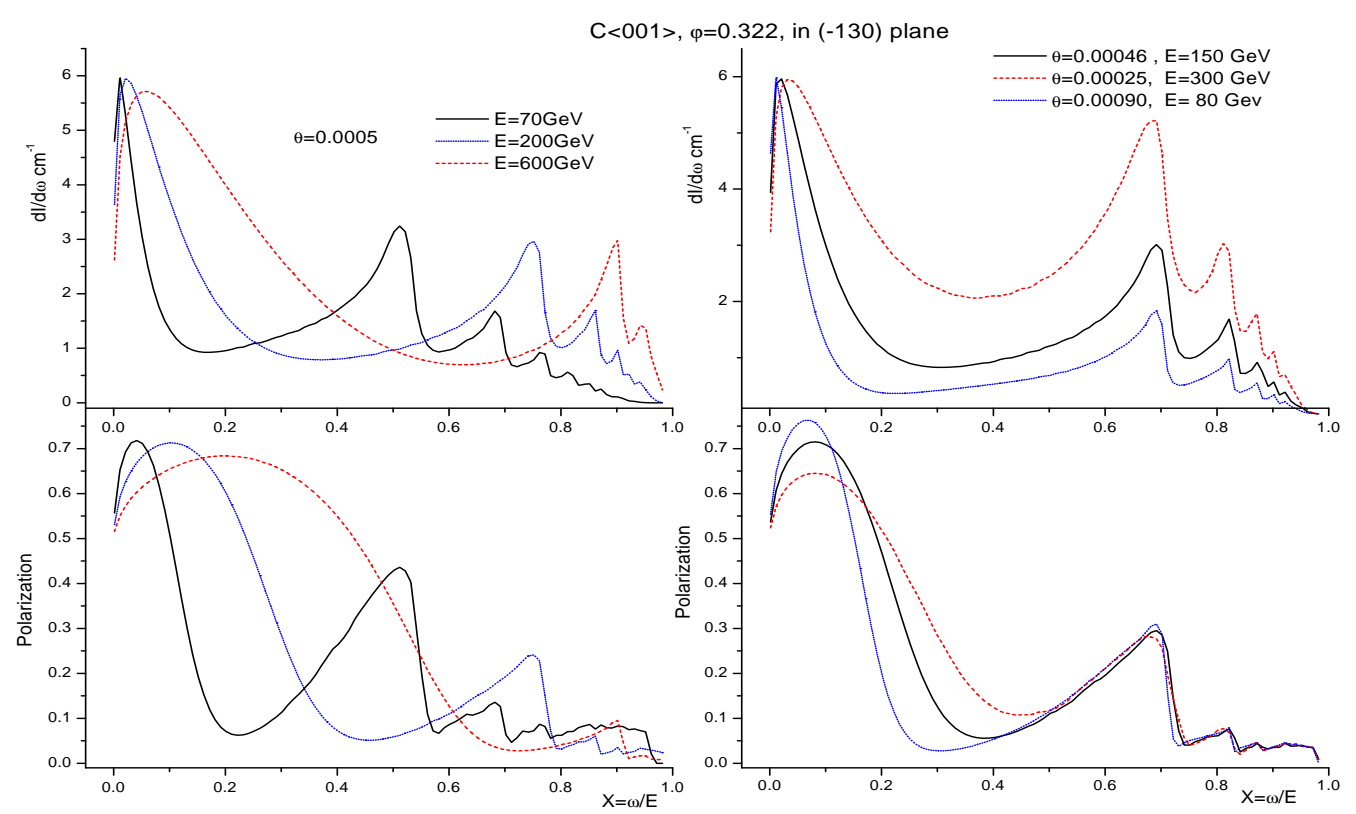

Fig.3: Energy dependence of spectral distributions of intensity and degree of polarization.

calculations and is far from pure magnetic bremstrahlung nature. It more resembles the modified coherent spectrum, with typical peaks and dips. That means that at these values of incident angles the functions in the sums $\sum_{\mathbf{q}}^{F}$ cannot be expanded in powers of $\left|q_{z}\right| \tau$ in all integration region, and together with magnetic bremstrahlung contribution, which comes 
from low $\tau$ region also contribute coherent effects which come from high $\tau$ region. Only at very small angles of incidence, about 3-5 times ( for $\varepsilon \sim 100-600 \mathrm{GeV}$ ) less than $\psi_{c}$, the soft part of spectrum become of pure magnetic bremstrahlung nature and could be described in BKS approximation. Note, that hard part of spectra is well described in BKS approximation starting from large values of incident angles (practically from $\vartheta \leq \vartheta_{v}$ ).
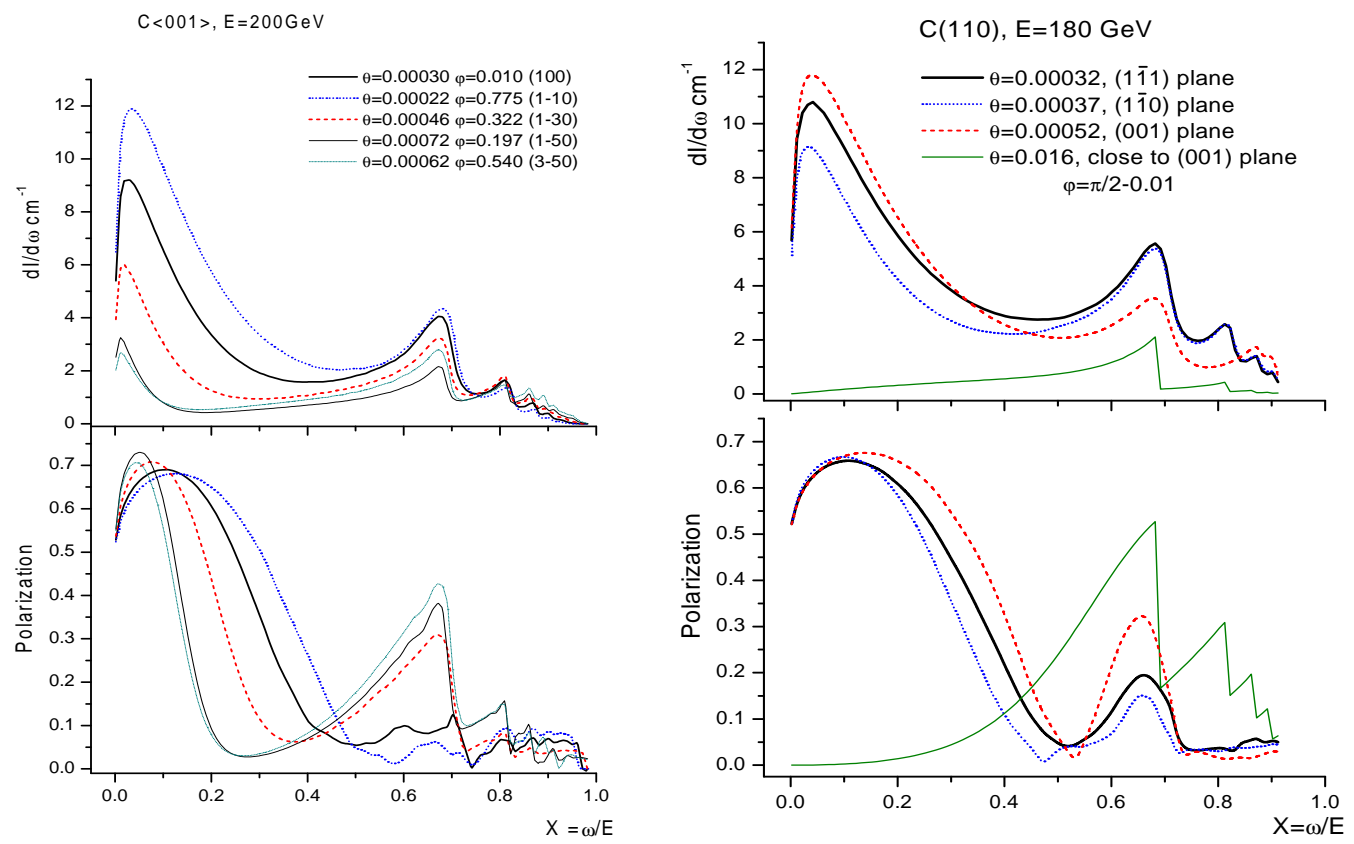

Fig.4: Spectral distributions of intensity and degree of polarization for different crystal orientations for fixed position of hard photon peak.

We also present large number of intensity and polarization spectra to demonstrate the dependence of angles, energies and crystal orientations and make it possible to find optimal configurations for producing high energy photon beams. Some of the analogous nonpolarized intensity spectra were already presented and analyzed in different papers [2-6]. The energy dependence at fixed incident angles is demonstrated in Fig.3a. Hard peaks in intensity spectra move to the left with increasing energy, slightly change their form, the heights of the peaks being practically independent of energy, corresponding peaks in polarization spectra also moved to the left and their heights decrease. Soft peak also moves to the left and becomes wider. On Fig.3b we demonstrate the energy dependence of spectra at different angles, chosen in such a way that first hard peak is situated at fixed value of $x \sim 2 / 3$. The height of hard peak in intensity spectra increase with increasing energy, the hard part of polarization spectra practically remain unchanged. So at very high energies it will be more and more preferable to use high frequency peaks in photon spectra for producing high energy photon beams. On Fig. 4 we present the spectra for $<001>$ and $<110>$ diamond crystal orientation when electron moves close to the main crystallographic planes at fixed energy. Angles are arranged in such a way that first hard peak in all spectra is situated at $x \sim 2 / 3$. The maximal height of intensity peak takes place for $<110>$ orientation when electron moves close to $(1 \overline{1} 1)$ or $(1 \overline{1} 0)$ planes and these configurations are the most preferable for production of nonpolarized high energy photons. The degree of polarization in hard photon peaks for these configurations appeared rather low. It is interesting that if we are going from strong 
planes to weak ones, the intensity of hard photon peak is decreasing and degree of polarization increasing. All these features could be qualitatively understood in modified coherent theory. At hard photon peaks the contributions of different planes are summed in nonpolarized photon intensity $\left(I_{0}\right)$, but in polarized photon intensities $\left(I_{1}, I_{3}\right)$ they could have opposite signs or one of the planes could not contribute. Therefore, the degree of polarization very strongly depend on crystal orientation. As one can see from Fig.4, for some orientations different contributions in polarized intensities practically compensate each other. It seems impossible to find crystal orientation, for which the contributions of all main plains will be summed in $I_{1}$ and $I_{3}$. Therefore, the maximal polarization for hard photon peak at given value of $x$ can be achieved for sufficiently large angles of incident electron to the crystallographic axis when hard photon peak appeared to be a typical coherent peak, corresponding to contribution of one plane and isn't affected by influence of even very weak planes. This is also demonstrated in Fig.5 We present the intensity and polarization spectra for $<001>$ diamond crystal for electron moving close to $(1 \overline{1} 0)$ plane for large $\theta$ and different $\varphi$, keeping the position of main peak fixed at $x \sim 2 / 3$. In wide range of $\varphi$, starting from $\varphi \sim \pi / 4$, height of the peak and degree of polarization remain practically unchanged and coincide with presented curves for $\varphi=\pi / 4-0.05$, if we keep electron far from neighboring planes. When we come closer to even rather weak planes, the height of the peak increases and the degree of polarization decreases. We demonstrate this effect for weak $(\overline{3} 50)$ plane $\left(U_{0} \sim 1.2 \mathrm{eV}\right)$. It is worth also noting that when we come close enough to such week plane, we could see even considerable magnetic bremstrahlung contribution. We have also checked this properties for weaker

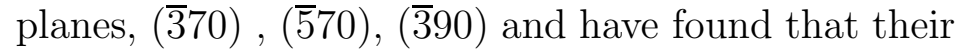
influence cannot be entirely ignored.

The most preferable crystal orientations for production of polarized high energy photons we find for $<001>$ oriented diamond crystal when electron moves close to $(1 \overline{1} 0)$ plane (for instance at $\theta=0.028$

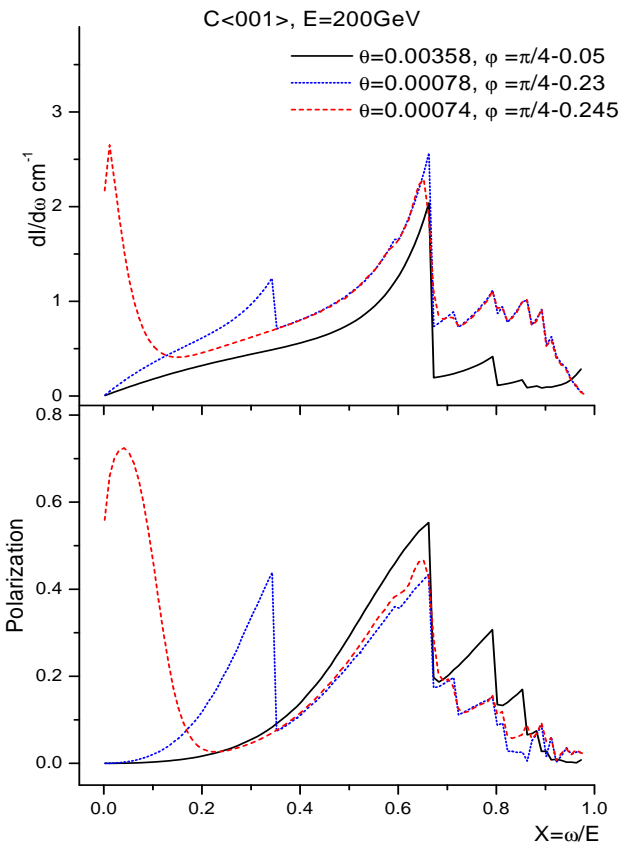

Fig.5: The influence of high order plane on intensity and polarization in coherent peak. $\operatorname{rad}, \varphi=0.735 \mathrm{rad}$ for $x \sim 2 / 3)$ and for $<110>$ oriented crystal when electron also moves close to $(1 \overline{1} 0)$ plane (for instance, at $\theta=0.028 \mathrm{rad}$, $\varphi=1.536 \mathrm{rad}$ for $x \sim 2 / 3$ ). For these configurations the maximal degree of polarization could be achieved for maximal intensities. These configurations bring exactly to the same results. The exact values of angles do not matter, one only should arrange angles to fix the main coherent peak at given value of $x$ and keep angles far from influence of neighboring planes.

This work was supported by the ISTC grant, project A-099. One of us (N.T) is grateful to Prof. R.Avakian for suggestion to participate in this project. 


\section{Appendix}

The function $F_{0}(z, \lambda)$ from Eqs.(9-11) can be expressed through generalized hypergeometric functions, ${ }_{p} F_{q}\left(\left(a_{p}\right) ;\left(b_{p}\right) ; x\right)$ :

$$
F_{0}(z, \lambda)=\frac{\pi}{6}+\frac{\pi z{ }_{1} F_{2}\left(\left(\frac{1}{3}\right) ;\left(\frac{2}{3}, \frac{4}{3}\right) ; \frac{z^{3}}{9 \lambda^{2}}\right)}{(3 \lambda)^{2 / 3} \Gamma\left(\frac{2}{3}\right)}-\frac{3^{\frac{1}{6}} z^{2} \Gamma\left(\frac{2}{3}\right){ }_{1} F_{2}\left(\left(\frac{2}{3}\right) ;\left(\frac{4}{3}, \frac{5}{3}\right) ; \frac{z^{3}}{9 \lambda^{2}}\right)}{4 \lambda^{4 / 3}}
$$

Functions $F_{1}(z, \lambda)$ and $F_{2}(z, \lambda)$ are expressed through combinations of Bessel functions of the first kind, $J_{\nu}(x)$, and of the third kind, $K_{\nu}(x)$ :

$$
\begin{aligned}
& F_{1}(z, \lambda)=\theta(z) \frac{\sqrt{z}}{\sqrt{3} \lambda} K_{\frac{1}{3}}\left(\frac{2 z^{3 / 2}}{3 \lambda}\right)+\theta(-z) \frac{\sqrt{-z}}{3 \lambda}\left(J_{\frac{1}{3}}\left(\frac{2(-z)^{3 / 2}}{3 \lambda}\right)+J_{-\frac{1}{3}}\left(\frac{2(-z)^{3 / 2}}{3 \lambda}\right)\right) \\
& F_{2}(z, \lambda)=\theta(z) \frac{z}{\sqrt{3} \lambda^{2}} K_{\frac{2}{3}}\left(\frac{2 z^{3 / 2}}{3 \lambda}\right)+\theta(-z) \frac{z}{3 \lambda^{2}}\left(J_{\frac{2}{3}}\left(\frac{2(-z)^{3 / 2}}{3 \lambda}\right)-J_{-\frac{2}{3}}\left(\frac{2(-z)^{3 / 2}}{3 \lambda}\right)\right)
\end{aligned}
$$

Here $\Gamma(x)$ is Euler gamma function and $\theta(x)$ is Heaviside function. Note, that:

$$
F_{1}(z, \lambda)=\frac{d F_{0}(z, \lambda)}{d z}, \quad F_{2}(z, \lambda)=-\frac{d F_{1}(z, \lambda)}{d z} .
$$

Instead of making use of existing programs for calculations of special functions, we have elaborated the calculation algorithm directly for functions $F_{i}(z, \lambda)$, connecting the asymptotic expansion in powers of $1 / z$ with low $z$ expansion in power of $z$. We present the corresponding equations only for $F_{0}(z, \lambda)$, analogous equations for $F_{2}(z, \lambda)$ and $F_{1}(z, \lambda)$ could be derived by differentiation or from corresponding expansions of Bessel functions. The asymptotic expansion of $F_{0}(z, \lambda)$ has the following form:

$$
\begin{aligned}
& F_{0}(z, \lambda)=\frac{\pi}{2}-\frac{1}{2} \exp \left(-\frac{2 z^{3 / 2}}{3 \lambda}\right) \sqrt{\frac{\pi \lambda}{z^{3 / 2}}}\left(1-\frac{41 \lambda}{48 z^{3 / 2}}+\frac{9241 \lambda^{2}}{4608 z^{3}}\right), \quad z \gg 1 \\
& F_{0}(z, \lambda)=-\frac{\pi}{2}+\sqrt{\frac{\pi \lambda}{(-z)^{3 / 2}}}\left[\left(1+\frac{9241 \lambda^{2}}{4608 z^{3}}\right) \cos \left(\frac{2(-z)^{3 / 2}}{3 \lambda}+\frac{\pi}{4}\right)+\right. \\
& \left.\frac{41 \lambda}{48(-z)^{3 / 2}} \sin \left(\frac{2(-z)^{3 / 2}}{3 \lambda}+\frac{\pi}{4}\right)\right], \quad \quad z \ll-1
\end{aligned}
$$

Power series expansion in powers of $z$ follows from definition of generalized hypergeometric functions:

$$
\begin{aligned}
F_{0}(z, \lambda)= & \frac{\pi}{6}+\frac{\pi z}{(3 \lambda)^{2 / 3} \Gamma\left(\frac{2}{3}\right)}\left[1+\sum_{n=1}^{\infty} \frac{t^{n}}{n !} \prod_{k=1}^{n} \frac{3 k-2}{(3 k-1)(3 k+1)}\right]- \\
& \frac{3^{\frac{1}{6}} z^{2} \Gamma\left(\frac{2}{3}\right)}{4 \lambda^{4 / 3}}\left[1+\sum_{n=1}^{\infty} \frac{t^{n}}{n !} \prod_{k=1}^{n} \frac{3 k-1}{(3 k+1)(3 k+2)}\right],
\end{aligned}
$$

where $t=z^{3} / 3 \lambda^{2}$. Asymptotic expansions (A.4-A.5) work with accuracy better than $0.1 \%$ starting from $|z| / \lambda^{2 / 3} \sim 4$ and Eqs. (A.4-A.5) and (A.6) could be connected at this point. One should take at this point about 10 terms of expansion (A.6) . Such calculation algorithm proved to be very effective and essentially reduces the computer calculation time. 


\section{References}

[1] M.L. Ter-Mikaelyan, High Energy Electromagnetic Processes on Condensed Media, Wiley-Intercience, New-York,1972.

[2] .N.Bayer, V.M. Katkov and V.M. Strakhovenko, Electromagnetic Processes at High Energies in Oriented Single Crystals, World Scientific, Singapore, 1998.

[3] V.N.Bayer, V.M. Katkov and V.M. Strakhavenko, Nucl.Instr. Methods. B69,(1992) 25.

[4] Yu.V.Kononets, I.S. Tupitsyn, Pisma Zh. Eksp. Teor. Fiz. 59, 491-497 (1994) 148-153.

[5] V.M. Strakhovenko, Nucl.Instr. and Meth. B145, 120 (1998).

[6] S.M.Darbinyan, N.L.Ter-Isaakyan, hep-ph/9809559, 1998; JETP lett.69,180, (1999). 